\title{
CMTCN: A web tool for investigating cancer-specific microRNA and transcription factor co-regulatory networks
}

Ruijiang Li ${ }^{1}$, Hebing Chen ${ }^{1}$, Shuai Jiang ${ }^{1}$, Wanying Li ${ }^{1}$, Hao Li ${ }^{1}$, Zhuo Zhang ${ }^{1}$, Hao Hong ${ }^{1}$, Xin Huang ${ }^{1}$, Chenghui Zhao ${ }^{1}$, Yiming Lu ${ }^{\text {Corresp., }}{ }^{1}$, Xiaochen Bo ${ }^{\text {Corresp. } 1}$

${ }^{1}$ Department of Biotechnology, Beijing Institute of Radiation Medicine, Beijing, China

Corresponding Authors: Yiming Lu, Xiaochen Bo

Email address: ylu.phd@gmail.com, boxc@bmi.ac.cn

Transcription factors (TFs) and microRNAs (miRNAs) are well-characterized trans-acting essential players in gene expression regulation. Growing evidence indicates that TFs and miRNAs can work cooperatively, and their dysregulation has been associated with many diseases including cancer. A unified picture of regulatory interactions of these regulators and their joint target genes would shed light on cancer studies. Although online resources developed to support probing of TF-gene and miRNA-gene interactions are available, online applications for miRNA-TF co-regulatory analysis, especially with a focus on cancers, are lacking. In light of this, we developed a web tool, namely CMTCN (freely available at www.cbportal.org/CMTCN), which constructs miRNA-TF co-regulatory networks and conducts comprehensive analyses within the context of particular cancer types. With its user-friendly provision of topological and functional analyses, CMTCN promises to be a reliable and indispensable web tool for biomedical studies. 
1 CMTCN: A web tool for investigating cancer-specific microRNA and transcription factor 2 co-regulatory networks

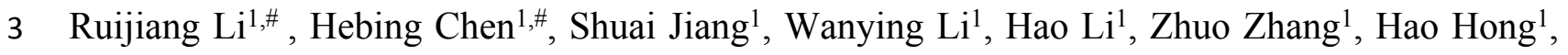
4 Xin Huang ${ }^{1}$, Chenghui Zhao ${ }^{1}$, Yiming $\mathrm{Lu}^{1}$ and Xiaochen $\mathrm{Bo}^{1}$

$5 \quad{ }^{1}$ Beijing Institute of Radiation Medicine, Beijing 100850, P.R.China

6 \# These authors contributed equally to this project as co-first authors

7

8

9 Corresponding authors:

10 Yiming $\mathrm{Lu}^{1}$ and Xiaochen $\mathrm{Bo}^{1}$

11 Email address: ylu.phd@gmail.com, boxc@bmi.ac.cn 


\begin{abstract}
12 Abstract
13 Transcription factors (TFs) and microRNAs (miRNAs) are well-characterized trans-acting 14 essential players in gene expression regulation. Growing evidence indicates that TFs and miRNAs 15 can work cooperatively, and their dysregulation has been associated with many diseases including 16 cancer. A unified picture of regulatory interactions of these regulators and their joint target genes 17 would shed light on cancer studies. Although online resources developed to support probing of 18 TF-gene and miRNA-gene interactions are available, online applications for miRNA-TF co19 regulatory analysis, especially with a focus on cancers, are lacking. In light of this, we developed 20 a web tool, namely CMTCN (freely available at www.cbportal.org/CMTCN), which constructs 21 miRNA-TF co-regulatory networks and conducts comprehensive analyses within the context of 22 particular cancer types. With its user-friendly provision of topological and functional analyses, 23 CMTCN promises to be a reliable and indispensable web tool for biomedical studies.
\end{abstract}




\section{Introduction}

Gene expression regulation is a complex biological process involving various regulators across multiple levels. Because it controls organism development and cell homeostasis (Yu et al. 2008), gene expression dysregulation is closely associated with disease processes. In gene expression regulation system, transcription factors (TFs) and microRNAs (miRNAs) have been recognized to play important roles at transcriptional level and post-transcriptional level respectively. Moreover, increasing evidence suggests that miRNAs and TFs are able to work together, mainly to buffer gene expression and/or adjust signaling (Bracken et al. 2016). Specifically, miRNAs and TFs have been shown to regulate shared target genes in feed-forward-loops (FFLs) and co-regulating pairs (Zhang et al. 2013). At the network level, miRNA-TF FFLs and co-regulating pairs are major network motifs (i.e. genetic interconnection patterns that occur more often by chance in biological networks), serving as basic building blocks of a complex regulatory system (Anastasiadou et al. 2017; Guo et al. 2016). Hence, perturbations of the interwoven regulatory patterns involving miRNAs and TFs trigger global alterations in gene expression and are associated with many diseases, including cancer.

Cancer is a complex, heterogeneous disease whose etiology involves diverse genetic and environmental factors. In the complex cancer-related gene expression regulation networks, miRNAs and TFs can work cooperatively as oncogenes or tumor suppressors (Yan et al. 2012). The construction and analysis of miRNA-TF co-regulatory networks may be used to improve our understanding of tumorigenesis and may suggest novel therapeutic targets. Indeed, analyses of FFLs and co-regulatory patterns have already revealed an essential role of their combined regulatory influence in some well-studied cancers. For example, in colorectal cancer, Wang et al. found that aberrant expression of two miRNAs (hsa-mir-25 and hsa-mir-31), one TF (BRCA1), and two other genes (ADAMTSL3 and AXIN1) affected patient survival, and thus provided clues regarding the components that determine colorectal cancer prognosis (Wang et al. 2017). Additionally, employing FFL detection and glioblastoma multiforme-specific co-regulatory network construction and analysis, Sun et al. discovered that the miRNA hsa-mir-34a plays a key role in glioblastoma multiforme, a lethal form of primary brain cancer (Sun et al. 2012). Likewise, employing miRNA-TF co-regulatory network analysis in breast cancer, Qin et al. found novel potential breast cancer driver genes (Qin et al. 2015).

Several web resources have been developed to unravel how miRNAs and TFs interact with 
genes, including resources for TF-gene regulation (Bovolenta et al. 2012; Han et al. 2017; Jiang et al. 2007; Zheng et al. 2008), and numerous tools for obtaining miRNA targets by experiments and predictions (Chou et al. 2017; Jiang et al. 2009; Wingender et al. 2000; Xiao et al. 2009; Yang et al. 2011). Although the identification of TF and miRNA targets is a key step in studying miRNATF co-regulation, there remains a need to combine these two forms of basic regulatory information together technically to enable identification of co-regulatory relationships and establish coregulatory networks. Although combining co-regulatory information with disease-related knowledgebases is critical for biomedical research, online tools based on these ideas are lacking.

Here, we report the design, development, and testing of an online application called CMTCN. CMTCN collects and integrates the published regulatory relationships among miRNAs, TFs, and target genes from 11 databases and provides a means of curating cancer-specific interactions by referring to documented cancer-related gene and miRNA databases. It conducts systematic explorations of major co-regulatory motifs, namely co-regulating pairs and FFLs that consist of miRNAs, TFs, and cancer-related genes. By identifying co-regulatory interactions, CMTCN can establish miRNA-TF co-regulatory networks for cancers and it provides useful analyses for understanding the molecular mechanisms underlying cancer pathogenesis.

\section{Materials and methods}

\section{Design and workflow}

CMTCN was developed by way of a five-step computational pipeline (Fig. 1). In step one, CMTCN utilized information provided by established regulatory databases of both predicted and experimentally validated interactions (Fig. 1A, Table S1). In total, 67,770 TF-gene, 177,724 TFmiRNA, 630,106 miRNA-gene, and 97,580 miRNA-TF interactions were collected. In step two, CMTCN curated cancer-related genes/miRNAs manually for 33 types of cancer by referring to cancer gene/miRNA databases, including TissGDB (Kim et al. 2017), SEGreg (Tang et al. 2018), IntOGen (Gonzalez-Perez et al. 2013), HMDD v2.0 (Li et al. 2014), miR2Disease (Jiang et al. 2009), PhenomiR (Ruepp et al. 2010), and miRCancer (Xie et al. 2013) (Fig. 1B, Table S2-S3). In step three, CMTCN screened out cancer-related regulatory interactions whose target nodes or regulator nodes are known to be relevant to cancer, forming an entirely synthetic network by pooling four types of interactions (Fig. 1C). Finally, in step four, CMTCN identified FFLs and coregulatory pairs from the combinatorial network using the network motif detection algorithm 
FANMOD (Wernicke \& Rasche 2006) and, in step five, constructed the co-regulatory network and incorporated expression data from The Cancer Genome Atlas (TCGA) (Katarzyna et al. 2015) to refine its discoveries (Fig. 1D-F).

The online CMTCN interface is a neat and user-friendly dashboard layout with two main modules: Start and Analysis. Users initiate their research in the 'Start' module with a three-step job submission process. After the job has been submitted, the webserver jumps to the 'Analysis' module where there is access to network-centric analysis, including a cancer-specific co-regulatory network display, network topology analysis, a co-regulatory interactions query, and enrichment analysis of genes and miRNAs in a co-regulatory network (Fig. 1G).

\section{Data input}

The user initiates a cancer-specific miRNA-TF co-regulation analysis through the construction of a co-regulatory network. CMTCN displays the co-regulatory network and provides detailed investigation for the network (Fig. 2).

The user begins in the 'Start' module with the following three steps: (i) choose a specific cancer; (ii) select a regulatory data source; and (iii) choose whether to analyze the full cancerspecific co-regulatory miRNA-TF network or to view the co-regulatory network for specific genes/miRNAs of interest (Fig. 2A). Currently, CMTCN supports 33 types of cancer. For coregulatory network construction, users are given the option of three evidence levels (validated, predicted, or both) and two angles (full co-regulation network or co-regulatory subgraph). When the full network is selected, CMTCN provides an overall co-regulatory network for a specific cancer. When the co-regulatory subgraph is selected, users can view the genes/miRNAs they are interested in. To facilitate analyses, CMTCN provides the gene sets associated with clinical stage (Lee et al. 2015) and high mutation rates (Sun et al. 2018) for each type of cancer. Users can input the genes they are interested in directly or upload a gene list in the form of a .txt file for query. The sample .txt file is provided in Data S1.

\section{Functionality of CMTCN}

\section{Identification of miRNA-TF co-regulatory interactions}

CMTCN pools TF-gene, TF-miRNA, miRNA-gene, miRNA-TF regulatory relationships. Based on these relationships, CMTCN focuses on co-regulatory pairs and three-node FFLs identified with the help of the network motif detection algorithm. A co-regulatory pair includes a TF and miRNA that regulate a gene simultaneously. There are three types of three-node FFLs: TF-FFLs, 
117 miRNA-FFLs, and composite-FFLs. In a TF-FFL, the TF is the master regulator, which regulates 118 a partner miRNA and their joint target. In a miRNA-FFL, the miRNA is the master regulator,

119

120

121

122

123

124

125

126

127

128

129

130

131

132

133

134

135

136

137

138

139

140

141

142

143

144

145

146

147

repressing its partner TF and their joint target gene. A TF-FFL and miRNA-FFL can combine to form a composite-FFL in which the miRNA and TF regulate each other. CMTCN can query and display each co-regulatory pattern in detail, and can incorporate expression data from TCGA to refine co-regulatory interactions (Fig. 2D).

\section{Co-regulatory interactions refinement}

CMTCN capitalizes on TCGA expression data to select important co-regulatory interactions. TCGA RNA-Seq data (run date 2016-01-28) provided in the Firehose data repository are accessed using the R package RTCGAToolbox (Samur 2014). CMTCN calculates pairwise Spearman correlation values between TFs, miRNAs, and genes. Users can refine co-regulatory pairs or FFLs on the basis of correlation p-values and correlation coefficients. For instance, when the user sets the p-value cutoff to 0.05 and the correlation coefficient cutoff to 0.2 , CMTCN displays a TFtarget edge of $p<0.05$ with correlation coefficients whose absolute values are $\geqslant 0.2$. Since most miRNAs are assumed to inhibit the expression of their targets (Beermann et al. 2016), CMTCN shows miRNA-target edge $\mathrm{p}<0.05$ and correlation coefficient $\leqslant-0.2$. Users can indicate which types of TF-target regulation they need. CMTCN gives the user the ability to select to differentiate between positive and negative TF regulation. Thus, if the user needs to examine only positive or negative regulation, $\mathrm{CMTCN}$ can retain only positive or negative correlation coefficient interactions, respectively.

\section{Network visualization}

CMTCN utilizes major co-regulatory motifs to form a cancer-specific miRNA-TF co-regulatory network. It uses the D3.js to depict an interactive and intuitive co-regulatory network map in which genes, TFs, and miRNAs are represented by yellow, red, and green nodes, respectively. To improve the presentation of the force-directed graph, users can adjust link distance, node repulsion, and the number of co-regulatory relationships displayed. CMTCN network graphs presented can be saved as images (Fig. 2B).

\section{Network topology analysis}

The key nodes in a co-regulation network have biological significance because they are signal convergence sites with pronounced control and influence over the network; accordingly, they represent potential candidates for biomarker prediction, clinical prognosis, and treatment (Barabási 
148

149

150

151

152

153

154

155

156

157

158

159

160

161

162

163

164

165

166

167

168

169

170

171

172

173

174

175

176

177

178

et al. 2011). CMTCN uses three indicators in its key node analysis: node degree, hub score, and authority score (Kleinberg 1999) (Fig. 2C). Node degree represents the number of edges that meet at a vertex. A node with a high hub score contains a large number of outgoing links, and a node with a high authority score is pointed to by many other nodes with high hub scores. Letting A be the adjacency matrix of the graph, the hub score is defined as the principal eigenvector of $\mathrm{AA}^{\mathrm{T}}$, and the authority score is the principal eigenvector of $\mathrm{A}^{\mathrm{T}} \mathrm{A}$. CMTCN uses appropriate pictures to produce a vivid presentation of scoring results.

\section{Gene/miRNA enrichment analysis}

To better capture and mine biological roles of a co-regulatory network, CMTCN takes advantage of annotated gene/miRNA sets from GSEA (Subramanian et al. 2005) and MiEEA (Backes et al. 2016), thereby enabling functional enrichment analysis of genes, TFs, and miRNAs involved in the co-regulatory network. CMTCN enables detailed gene-ontology association analyses with a variety of biological and biomedical ontologies, extending beyond GO (Consortium et al. 2000) and KEGG (Kanehisa \& Goto 2000), thereby providing clues for follow-up studies (Fig. 2E).

\section{Implementation}

The CMTCN website can be accessed freely and readily by all users without a login requirement. It supports most prevalent web browsers, including Google Chrome, Mozilla Firefox, Safari, and Internet Explorer (10 or later). It adjusts automatically to the layout of particular browsers and device types, from desktop computers to tablets and smart phones. CMTCN was written almost entirely in R code based on the R-Shiny web framework (Chang et al. 2014) and has been deployed on an Aliyun server. The backend database is implemented with SQLite (version 3.8.8.2).

\section{Results}

Functional use case of CMTCN

To better illustrate the functionality and utility of CMTCN, we studied the miRNA-TF coregulation of two specific cancers, namely thyroid carcinoma (THCA) and ovarian cancer (OV).

\section{Uncovering and analyzing the miRNA-TF co-regulatory network in THCA}

THCA is a common endocrine malignancy with an increasing worldwide incidence (Cabanillas et al. 2016). In CMTCN, we chose the THCA cancer set, selected the validated regulation information confidence level, and built a full miRNA-TF co-regulatory network for THCA. For each type of co-regulatory pattern, we required a p-value $<0.05$, an absolute value of correlation 
179

180

181

182

183

184

185

186

187

188

189

190

191

192

193

194

195

196

197

198

199

200

201

202

203

204

205

206

207

208

209

coefficient $\geq 0.2$, and both types of TF regulation. CMTCN established a THCA-specific miRNATF co-regulatory network comprised of 391 nodes and 518 links, with 710 co-regulatory pairs, 7 TF-FFLs, 1 miRNA-FFLs, and 2 composite-FFLs.

CMTCN then used network topology analysis to reveal the top-five genes in terms of authority score (MELK, PIGR, SNX5, CLU, and DAPK2) (Table S4). A comprehensive literature review of these genes confirmed their implicated roles in cancer diagnosis and therapy. MELK has been reported to be potential therapeutic targets for malignancies (Pitner et al. 2017). PIGR has the potential to be a candidate prognostic biomarker (Fristedt et al. 2014). Regulation of CLU by oncogenes and epigenetic factors has important consequences for mammalian tumorigenesis (Sala et al. 2009). The aberrant methylation, and hence silencing, of DAPK2 has been reported to play a critical role in thyroid cancer tumorigenesis and progression (Hu et al. 2006). Finally, reduced expression of SNX5 was shown recently to be related to promotion of thyroid tumorigenesis (Jitsukawa et al. 2017) and SNX5 expression studies can be used to support a pathology diagnosis of thyroid cancer (Ara et al. 2012). Additionally, CMTCN carried out a functional enrichment analysis for genes and TFs in the THCA-specific miRNA-TF coregulatory network. With KEGG pathway enrichment, CMTCN found 11 significant pathways, all of which were related to cancer (Table S5). CMTCN pinpointed four TFs (E2F4, TFDP1, $\mathrm{SP} 1, \mathrm{MYC}$ ) and one gene ACVR1 in the transforming growth factor- $\beta$ signaling pathway, a negative regulator of thyroid follicular cell growth (Geraldo et al. 2012).

\section{MiRNA-TF co-regulatory subnetwork of top mutated genes in $\mathrm{OV}$}

OV is highly aggressive gynecological cancer (Sung et al. 2017). We used CMTCN to establish an OV-specific miRNA-TF subnetwork encompassing the top-100 mutated genes in OV. Again, we set the confidence level to validate and required a $p$-value $<0.05$, an absolute correlation coefficient $\geq 0.2$ and both types of TF regulation. Our goal was to use CMTCN to reveal the miRNAs and TFs related to the top mutated genes in OV, as well as the regulatory effects of these miRNAs and TFs.

We obtained six co-regulated pairs and one TF-FFL related to the top-100 mutated genes in OV, which revealed six miRNAs and three TFs with possible associations with these top mutated genes. The sole TF-FFL obtained was comprised of a TF (TP53), a miRNA (hsa-mir-29c), and a joint target gene (PTEN). In this TF-FFL, the TF regulates both the miRNA and the target gene, with the miRNA repressing the target gene. Regarding OV pathogenesis, the loss function of 
210 PTEN, together with TP53 alteration is a common event (Martins et al. 2014). Interestingly, hsa211 mir-29c, an effector of regulator TP53, can also suppress cancer development (Li et al. 2018). The 212 possibility that abnormal expression of the two cross-talking regulators and their co-target gene 213 may be predictive of OV risk is worth further careful study in future experiments.

\section{Discussion}

The results of these demonstration studies, described before, show that CMTCN is able to uncover and analyze miRNA-TF co-regulation networks in a manner that can enhance our understanding of miRNA-TF gene regulatory mechanisms in different types of cancer and provide valuable information for cancer prognosis and therapy.

CMTCN explored miRNA-TF co-regulatory pairs and FFLs systematically and in a contextspecific manner. To enhance the power and accuracy of the discovery, CMTCN provides TCGA expression-based filtering options for calculations of pairwise correlations between miRNAs, TFs, and genes. Owing to its simplicity and large-scale network computing capability, like other related analyzation methods (Qin et al. 2015; Wang et al. 2017), CMTCN uses pairwise correlations to refine co-regulation. In addition to the refinement, CMTCN combines network topology information with co-regulatory relationship queries to provide a sum of degree, hub, and authority scores for each co-regulation interaction type, which supports the discovery of high-value coregulatory interactions. In fact, there are multiple ways to deal with co-regulatory interaction mining outcomes and there are opportunities to improve the co-regulatory analysis framework in future work. Methods, such as partial correlation or the emerging detrended partial-crosscorrelation analysis (DPCCA) method (Yuan et al. 2015), could be applied in the refinement step. Moreover, integrating our miRNA-TF co-regulatory network with other functional networks will potentiate the findings at a systems level.

\section{Conclusions}

Here, we introduced CMTCN as a user-friendly online tool for miRNA-TF co-regulation analysis in the context of cancer research. CMTCN characterized and detected co-regulatory pairs and three types of FFLs for each type of cancer. It constructed detailed and dynamic cancer-specific miRNATF co-regulatory networks that elucidate the interwoven pivotal roles of TFs, genes, and miRNAs 
241 be investigated further experimentally as potential biomarkers or drug targets. The program 242 supports various enrichment analyses for discovery of network gene/miRNA ontology 243 associations. Though it was developed for miRNA-TF co-regulation analysis studies specifically, 244 CMTCN has broad biomedical applications and can be utilized by cancer researchers as well as 245 systems biologists and epigenetic scholars. Cancer researchers can utilize CMTCN to find 246 candidate cancer genes; systems biologists can explore the qualities of the comprehensive 247 network-centric analyses of CMTCN. Epigeneticists can use CMTCN to interpret the integrative 248 global effects

of

TFs

and

miRNAs

on

cancer. 
249

250

251

252

253

254

255

256

257

258

259

260

261

262

263

264

265

266

267

268

269

270

271

272

273

274

275

276

277

278

279

280

281

282

283

284

285

286

287

288

289

\section{References}

Anastasiadou E, Jacob LS, and Slack FJ. 2017. Non-coding RNA networks in cancer. Nature Reviews Cancer 18:5-18. 10.1038/nrc.2017.99 DOI: 10.1038/nrc.2017.99.

Ara S, Kikuchi T, Matsumiya H, Kojima T, Kubo T, Ye RC, Sato A, Kon SI, Honma T, and Asakura K. 2012. Sorting nexin 5 of a new diagnostic marker of papillary thyroid carcinoma regulates Caspase-2. Cancer Science 103:1356-1362 DOI: 10.1111/j.13497006.2012.02296.x.

Backes C, Khaleeq QT, Meese E, and Keller A. 2016. miEAA: microRNA enrichment analysis and annotation. Nucleic Acids Research 44:W110-W116 DOI: 10.1093/nar/gkw345.

Barabási AL, Gulbahce N, and Loscalzo J. 2011. Network medicine: a network-based approach to human disease. Nature Reviews Genetics 12:56-68 DOI: 10.1038/nrg2918.

Beermann J, Piccoli MT, Viereck J, and Thum T. 2016. Non-coding RNAs in Development and Disease: Background, Mechanisms, and Therapeutic Approaches. Physiological Reviews 96:1297 DOI: 10.1152/physrev.00041.2015.

Bovolenta LA, Acencio ML, and Lemke N. 2012. HTRIdb: an open-access database for experimentally verified human transcriptional regulation interactions. Bmc Genomics 13:405 DOI: $10.1186 / 1471-2164-13-405$.

Bracken CP, Scott HS, and Goodall GJ. 2016. A network-biology perspective of microRNA function and dysfunction in cancer. Nat Rev Genet 17:719-732 DOI: 10.1038/nrg.2016.134.

Cabanillas ME, Mcfadden DG, and Durante C. 2016. Thyroid cancer. Lancet 388:2783 DOI: 10.1016/S0140-6736(16)30172-6.

Chang W, Cheng J, Allaire JJ, Xie Y, and Mcpherson J. 2014. Web Application Framework for $R$ : SpellPress.

Chou CH, Shrestha S, Yang CD, Chang NW, Lin YL, Liao KW, Huang WC, Sun TH, Tu SJ, and Lee WH. 2017. miRTarBase update 2018: a resource for experimentally validated microRNA-target interactions. Nucleic Acids Research 46 DOI: 10.1093/nar/gkx1067.

Consortium TGO, Ashburner M, Ball CA, Blake JA, Botstein D, Butler H, Cherry JM, Davis AP, Dolinski K, and Dwight SS. 2000. Gene Ontology: tool for the unification of biology. Nature Genetics 25:25-29 DOI: 10.1038/75556.

Fristedt R, Gaber A, Hedner C, Nodin B, Uhlén M, Eberhard J, and Jirström K. 2014. Expression and prognostic significance of the polymeric immunoglobulin receptor in esophageal and gastric adenocarcinoma. Journal of Translational Medicine 12:83 DOI: $\underline{10.1186 / 1479-}$ 5876-12-83.

Geraldo MV, Yamashita AS, and Kimura ET. 2012. MicroRNA miR-146b-5p regulates signal transduction of TGF- $\hat{I}^{2}$ by repressing SMAD4 in thyroid cancer. Oncogene 31:1910 DOI: 10.1038/onc.2011.381.

Gonzalez-Perez A, Perez-Llamas C, Deu-Pons J, Tamborero D, Schroeder MP, Jene-Sanz A, Santos A, and Lopez-Bigas N. 2013. IntOGen-mutations identifies cancer drivers across tumor types. Nat Methods 10:1081-1082 DOI: 10.1038/nmeth.2642.

Guo Y, Alexander K, Clark AG, Grimson A, and Yu H. 2016. Integrated network analysis 
290

291

292

293

294

295

296

297

298

299

300

301

302

303

304

305

306

307

308

309

310

311

312

313

314

315

316

317

318

319

320

321

322

323

324

325

326

327

328

329

330

reveals distinct regulatory roles of transcription factors and microRNAs. RNA 22:16631672 DOI: 10.1261/rna.048025.114.

Han H, Cho JW, Lee S, Yun A, Kim H, Bae D, Yang S, Kim CY, Lee M, and Kim E. 2017. TRRUST v2: an expanded reference database of human and mouse transcriptional regulatory interactions. Nucleic Acids Research 46 DOI: 10.1093/nar/gkx1013.

Hu S, Liu DR, Carson KA, Rosenbaum E, Cohen Y, Holt EH, Kiseljak VK, Rhoden KJ, Tolaney S, and Condouris S. 2006. Association of aberrant methylation of tumor suppressor genes with tumor aggressiveness and BRAF mutation in papillary thyroid cancer. International Journal of Cancer 119:2322-2329 DOI: 10.1002/ijc.22110.

Jiang C, Xuan Z, Zhao F, and Zhang MQ. 2007. TRED: a transcriptional regulatory element database, new entries and other development. Nucleic Acids Research 35:D137-D140 DOI: $10.1093 / \mathrm{nar} / \mathrm{gkl1041}$.

Jiang Q, Wang Y, Hao Y, Juan L, Teng M, Zhang X, Li M, Wang G, and Liu Y. 2009. miR2Disease: a manually curated database for microRNA deregulation in human disease. Nucleic Acids Research 37:D98-104 DOI: 10.1093/nar/gkn714.

Jitsukawa S, Kamekura R, Kawata K, Ito F, Sato A, Matsumiya H, Nagaya T, Yamashita K, Kubo T, and Kikuchi T. 2017. Loss of sorting nexin 5 stabilizes internalized growth factor receptors to promote thyroid cancer progression. Journal of Pathology 243 DOI: 10.1002/path.4951.

Kanehisa M, and Goto S. 2000. KEGG: Kyoto Encyclopedia of Genes and Genomes. Nucleic Acids Research 27:29-34.

Katarzyna T, Patrycja C, and Maciej W. 2015. The Cancer Genome Atlas (TCGA): an immeasurable source of knowledge. Contemporary Oncology 19:68-77 DOI: 10.5114/wo.2014.47136.

Kim P, Park A, Han G, Sun H, Jia P, and Zhao Z. 2017. TissGDB: tissue-specific gene database in cancer. Nucleic Acids Research DOI: 10.1093/nar/gkx850.

Kleinberg JM. 1999. Authoritative Sources in a Hyperlinked Environment. Journal of the Acm 46:604-632.

Lee H, Palm J, Grimes SM, and Ji HP. 2015. The Cancer Genome Atlas Clinical Explorer: a web and mobile interface for identifying clinical-genomic driver associations. Genome Medicine, 7,1(2015-10-27) 7:1-14 DOI: 10.1186/s13073-015-0226-3.

Li W, Yi J, Zheng X, Liu S, Fu W, Ren L, Li L, Hoon DSB, Wang J, and Du G. 2018. miR-29c plays a suppressive role in breast cancer by targeting the TIMP3/STAT1/FOXO1 pathway. Clin Epigenetics 10:64 DOI: 10.1186/s13148-018-0495-y.

Li Y, Qiu C, Tu J, Geng B, Yang J, Jiang T, and Cui Q. 2014. HMDD v2.0: a database for experimentally supported human microRNA and disease associations. Nucleic Acids Research 42:D1070 DOI: 10.1093/nar/gkt1023.

Martins FC, Santiago I, Trinh A, Xian J, Guo A, Sayal K, Jimenez-Linan M, Deen S, Driver K, Mack M, Aslop J, Pharoah PD, Markowetz F, and Brenton JD. 2014. Combined image and genomic analysis of high-grade serous ovarian cancer reveals PTEN loss as a common driver event and prognostic classifier. Genome Biol 15:526 DOI: 
10.1186/s13059-014-0526-8.

Pitner MK, Taliaferro JM, Dalby KN, and Bartholomeusz C. 2017. MELK: a potential novel therapeutic target for TNBC and other aggressive malignancies. Expert Opin Ther Targets 21 DOI: $10.1080 / 14728222.2017 .1363183$.

Qin S, Ma F, and Chen L. 2015. Gene regulatory networks by transcription factors and microRNAs in breast cancer. Bioinformatics 31:76-83 DOI: 10.1093/bioinformatics/btu597.

Ruepp A, Kowarsch A, Schmidl D, Buggenthin F, Brauner B, Dunger I, Fobo G, Frishman G, Montrone C, and Theis FJ. 2010. PhenomiR: a knowledgebase for microRNA expression in diseases and biological processes. Genome Biol 11:R6 DOI: 10.1186/gb-2010-11-1-r6.

Sala A, Bettuzzi S, Pucci S, Chayka O, Dews M, and Thomas-Tikhonenko A. 2009. Regulation of CLU Gene Expression by Oncogenes and Epigenetic Factors : Implications for Tumorigenesis. Advances in Cancer Research 105:115-132 DOI: 10.1016/S0065230X(09)05007-6.

Samur MK. 2014. RTCGAToolbox: A New Tool for Exporting TCGA Firehose Data. Plos One 9:e106397 DOI: 10.1371/journal.pone.0106397.

Subramanian A, Tamayo P, Mootha VK, Mukherjee S, Ebert BL, Gillette MA, Paulovich A, Pomeroy SL, Golub TR, Lander ES, and Mesirov JP. 2005. Gene set enrichment analysis: A knowledge-based approach for interpreting genome-wide expression profiles. Proceedings of the National Academy of Sciences 102:15545-15550 DOI: 10.1073/pnas.0506580102.

Sun J, Gong X, Purow B, and Zhao Z. 2012. Uncovering MicroRNA and Transcription Factor Mediated Regulatory Networks in Glioblastoma. PLoS Comput Biol 8:e1002488 DOI: 10.1371/journal.pcbi.1002488.

Sun Q, Li M, and Wang X. 2018. The Cancer Omics Atlas: an integrative resource for cancer omics annotations. Bmc Medical Genomics 11:63 DOI: 10.1186/s12920-018-0381-7.

Sung HY, Yang SD, Ju W, and Ahn JH. 2017. Aberrant epigenetic regulation of GABRP associates with aggressive phenotype of ovarian cancer. Experimental \& Molecular Medicine 49 DOI: $10.1038 / \mathrm{emm} .2017 .62$.

Tang Q, Zhang Q, Lv Y, Miao YR, and Guo AY. 2018. SEGreg: a database for human specifically expressed genes and their regulations in cancer and normal tissue. Briefings in Bioinformatics:1-7 DOI: 10.1093/bib/bbx173.

Wang H, Luo J, Liu C, Niu H, Wang J, Liu Q, Zhao Z, Xu H, Ding Y, Sun J, and Zhang Q. 2017. Investigating MicroRNA and transcription factor co-regulatory networks in colorectal cancer. BMC Bioinformatics 18:388 DOI: 10.1186/s12859-017-1796-4.

Wernicke S, and Rasche F. 2006. FANMOD: a tool for fast network motif detection. Bioinformatics 22:1152-1153 DOI: 10.1093/bioinformatics/bt1038.

Wingender E, Chen X, Hehl R, Karas H, Liebich I, Matys V, Meinhardt T, Prüß M, Reuter I, and Schacherer F. 2000. TRANSFAC: an integrated system for gene expression regulation. Nucleic Acids Research 28:316-319.

Xiao F, Zuo Z, Cai G, Kang S, Gao X, and Li T. 2009. miRecords: an integrated resource for 
372

373

374

375

376

377

378

379

380

381

382

383

384

385

386

387

388

389

390

391

392

393

394

395

microRNA-target interactions. Nucleic Acids Research 37:D105 DOI: 10.1093/nar/gkn851.

Xie B, Ding Q, Han H, and Wu D. 2013. miRCancer: a microRNA-cancer association database constructed by text mining on literature. Bioinformatics 29:638-644 DOI: 10.1093/bioinformatics/btt014.

Yan Z, Shah PK, Amin SB, Samur MK, Huang N, Wang X, Misra V, Ji H, Gabuzda D, and Li C. 2012. Integrative analysis of gene and miRNA expression profiles with transcription factor-miRNA feed-forward loops identifies regulators in human cancers. Nucleic Acids Res 40:e135 DOI: 10.1093/nar/gks395.

Yang JH, Li JH, Shao P, Zhou H, Chen YQ, and Qu LH. 2011. starBase: a database for exploring microRNA-mRNA interaction maps from Argonaute CLIP-Seq and Degradome-Seq data. Nucleic Acids Res 39:D202-209 DOI: 10.1093/nar/gkq1056.

Yu X, Lin J, Zack DJ, Mendell JT, and Qian J. 2008. Analysis of regulatory network topology reveals functionally distinct classes of microRNAs. Nucleic Acids Res 36:6494-6503 DOI: $10.1093 / \mathrm{nar} / \mathrm{gkn} 712$.

Yuan N, Fu Z, Zhang H, Lin P, Xoplaki E, and Luterbacher J. 2015. Detrended Partial-CrossCorrelation Analysis: A New Method for Analyzing Correlations in Complex System. Scientific Reports 5:8143 DOI: 10.1038/srep08143.

Zhang HM, Kuang S, Xiong X, Gao T, Liu C, and Guo AY. 2013. Transcription factor and microRNA co-regulatory loops: important regulatory motifs in biological processes and diseases. Briefings in Bioinformatics 16:45 DOI: 10.1093/bib/bbt085.

Zheng G, Tu K, Yang Q, Xiong Y, Wei C, Xie L, Zhu Y, and Li Y. 2008. ITFP: an integrated platform of mammalian transcription factors. Bioinformatics 24:2416-2417 DOI: 10.1093/bioinformatics/btn439. 


\section{Figure 1}

Overview of the CMTCN workflow.

(A) CMTCN utilized information provided by established regulatory databases of both predicted and experimentally validated interactions. (B) CMTCN curated cancer-related genes and miRNAs for 33 types of cancers by referring to established cancer genes/miRNAs databases. (C) CMTCN screened out cancer-related regulatory interactions whose target nodes or regulator nodes are known to be relevant to cancer, forming an entirely synthetic network by pooling four types of interactions. (D) CMTCN identified FFLs and co-regulatory pairs from the combinatorial network using a network motif detection algorithm. (E) By identifying co-regulatory interactions, CMTCN can establish miRNA-TF co-regulatory networks in different cancers. (F) CMTCN incorporated expression data from TCGA to refine discoveries. (G) CMTCN supports enriched network-centric downstream analysis, including cancer-specific co-regulatory network displays, network topology analyses, co-regulatory interactions queries, and intra-co-regulatory network gene/miRNA enrichment analyses. 

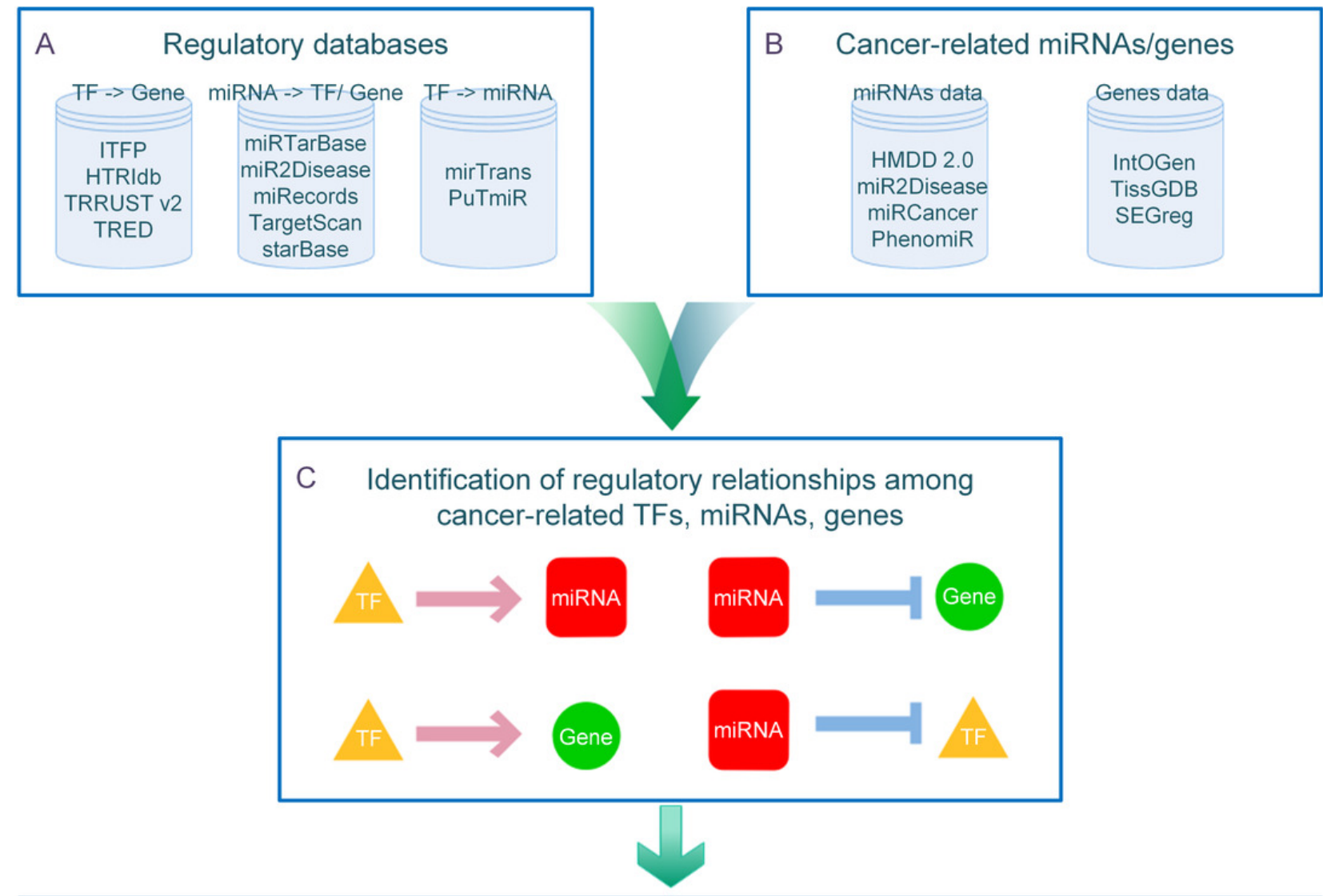

D

Detection of miRNA-TF co-regulatory motifs

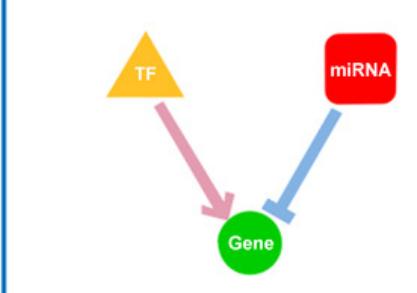

Co-regulatory pair

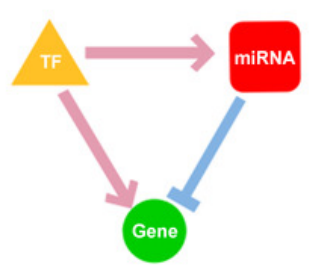

TF-FFL

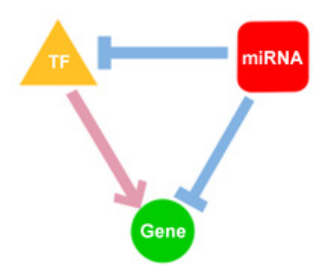

miRNA-FFL

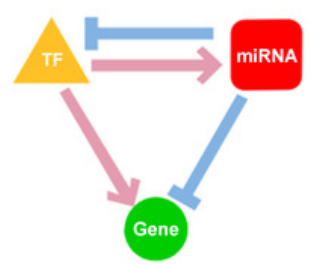

Composite-FFL

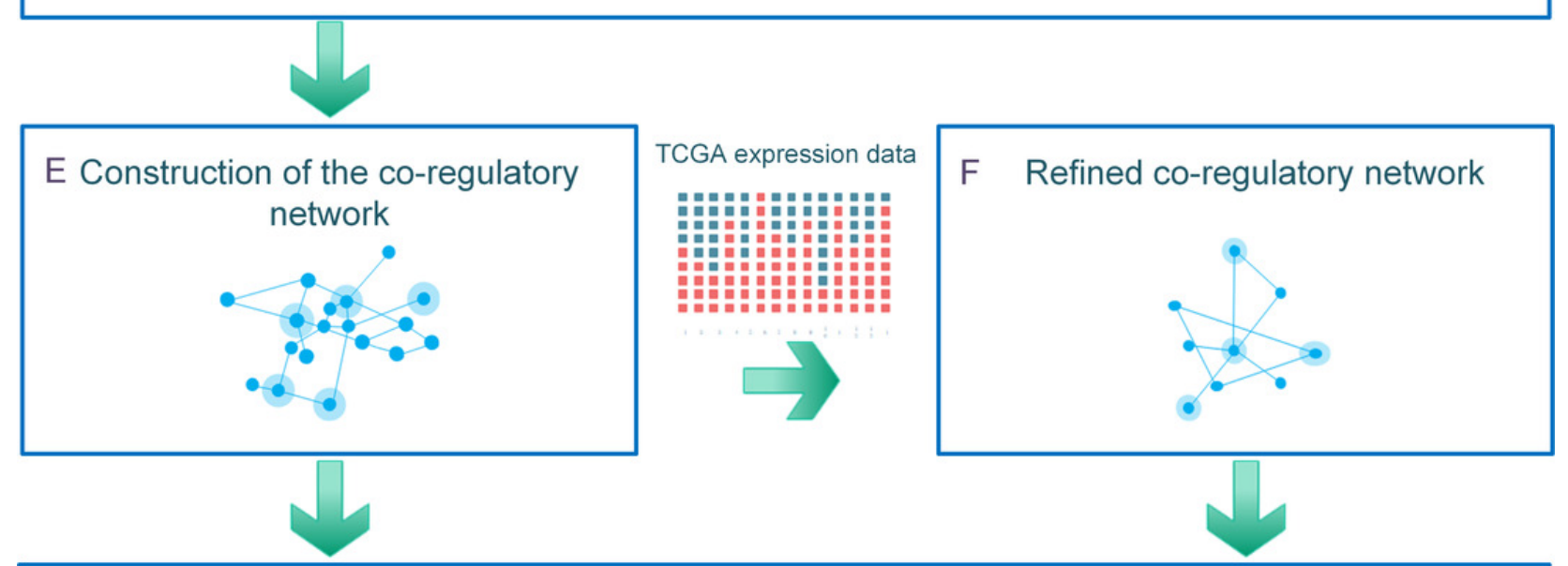

G

Downstream analysis
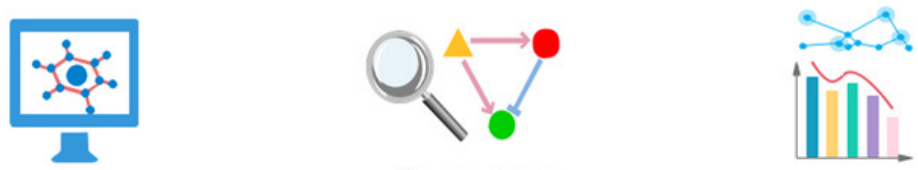

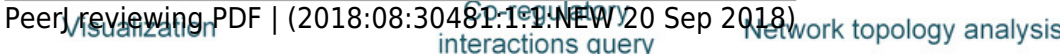

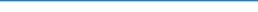




\section{Figure 2}

Features of the interactive CMTCN web service.

(A) Users initiate their study by three steps. First, the user selects an CMTCN-supported cancer type (currently, 33 to choose from), selects the desired evidence levels, and selects whether they want to study an entire co-regulation network or a subnet of co-regulatory network for genes of interest. (B) CMTCN displays an interactive and intuitive force network map for the co-regulatory network. (C) CMTCN uses three indicators to analyze the key nodes of the established co-regulatory network. (D) CMTCN can query each co-regulatory interaction type. (E) CMTCN makes functional enrichment analysis for genes, TFs, and miRNAs involved in the co-regulatory network. 


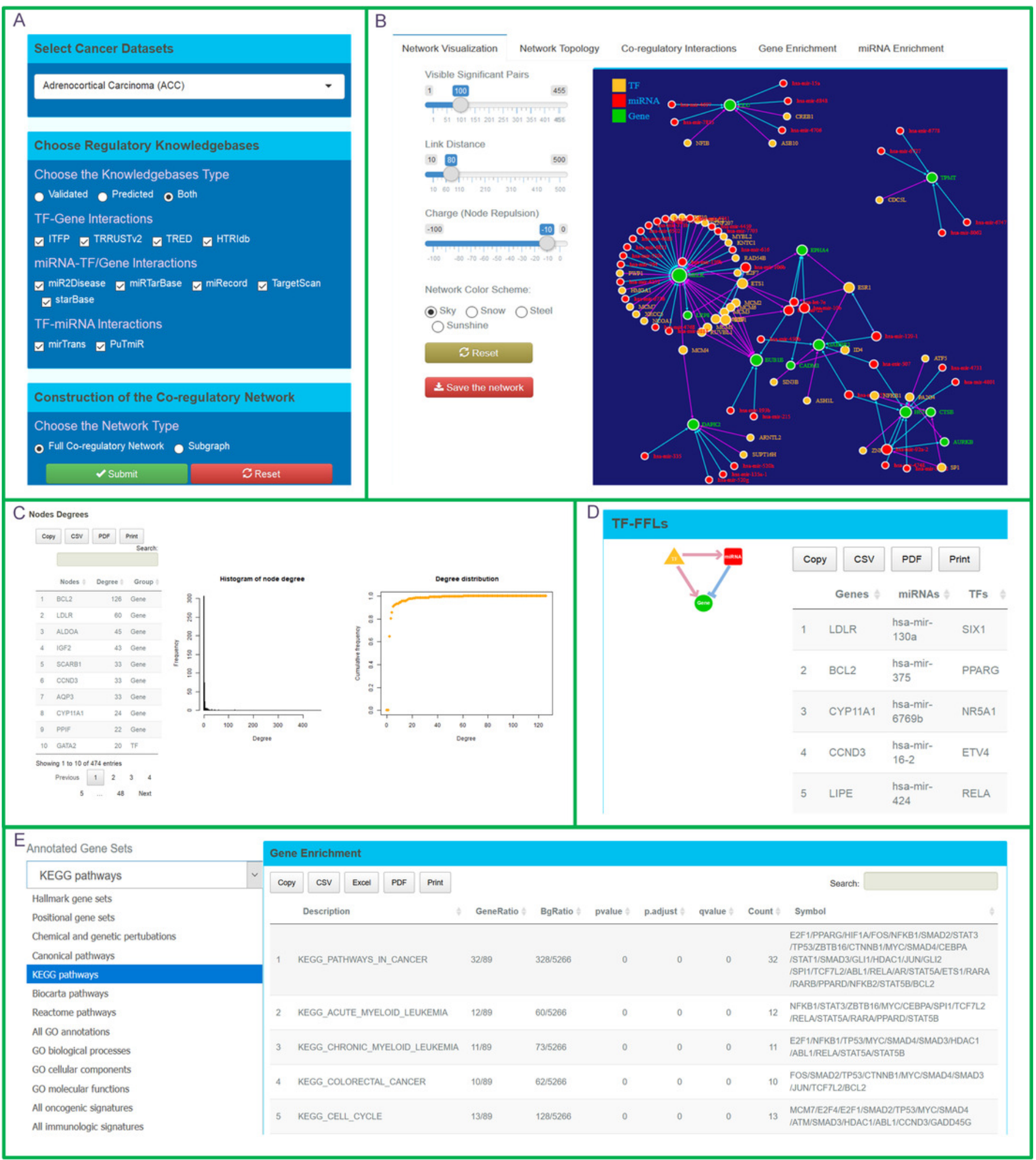

